\title{
THE MASSIVE PROGENITOR OF THE TYPE II-LINEAR SUPERNOVA 2009kr*
}

\author{
Nancy Elias-Rosa ${ }^{1}$, Schuyler D. Van Dyk ${ }^{1}$, Weidong Li ${ }^{2}$, Adam A. Miller ${ }^{2}$, Jeffrey M. Silverman ${ }^{2}$, \\ Mohan Ganeshalingam ${ }^{2}$, Andrew F. Boden ${ }^{3}$, Mansi M. Kasliwal ${ }^{3}$, József Vinkó ${ }^{4,5}$, Jean-Charles Cuillandre ${ }^{6}$, \\ Alexei V. Filippenko ${ }^{2}$, Thea N. Steele ${ }^{2}$, Joshua S. Bloom ${ }^{2}$, Christopher V. Griffith ${ }^{2}$, Io K. W. Kleiser ${ }^{2}$, \\ AND RYAN J. FOLEY ${ }^{7,8}$ \\ ${ }^{1}$ Spitzer Science Center, California Institute of Technology, 1200 East California Boulevard, Pasadena, CA 91125, USA; nelias@ipac.caltech.edu \\ ${ }^{2}$ Department of Astronomy, University of California, Berkeley, CA 94720-3411, USA \\ ${ }^{3}$ Division of Physics, Math, and Astronomy, California Institute of Technology, Pasadena, CA 91125, USA \\ ${ }^{4}$ Department of Optics and Quantum Electronics, University of Szeged, Dóm tér 9, Szeged H-6720, Hungary \\ ${ }^{5}$ Department of Astronomy, University of Texas, Austin, TX 78712, USA \\ ${ }^{6}$ Canada-France-Hawaii Telescope Corporation, 65-1238 Mamalahoa Hwy, Kamuela, HI 96743, USA \\ ${ }^{7}$ Harvard/Smithsonian Center for Astrophysics, 60 Garden Street, Cambridge, MA 02138, USA \\ Received 2009 December 16; accepted 2010 April 2; published 2010 April 16
}

\begin{abstract}
We present early-time photometric and spectroscopic observations of supernova (SN) 2009kr in NGC 1832. We find that its properties to date support its classification as Type II-linear (SN II-L), a relatively rare subclass of corecollapse supernovae ( $\mathrm{SNe}$ ). We have also identified a candidate for the $\mathrm{SN}$ progenitor star through comparison of pre-explosion, archival images taken with WFPC2 on board the Hubble Space Telescope with SN images obtained using adaptive optics plus NIRC2 on the $10 \mathrm{~m}$ Keck-II telescope. Although the host galaxy's substantial distance $(\sim 26 \mathrm{Mpc})$ results in large uncertainties in the relative astrometry, we find that if this candidate is indeed the progenitor, it is a highly luminous $\left(M_{V}^{0}=-7.8 \mathrm{mag}\right)$ yellow supergiant with initial mass $\sim 18-24 M_{\odot}$. This would be the first time that an SN II-L progenitor has been directly identified. Its mass may be a bridge between the upper initial mass limit for the more common Type II-plateau SNe and the inferred initial mass estimate for one Type II-narrow SN.
\end{abstract}

Key words: galaxies: individual (NGC 1832) - stars: evolution - supernovae: general - supernovae: individual (SN 2009kr)

\section{INTRODUCTION}

It is not yet exactly clear how to map massive stars of a given mass range to a core-collapse supernova (CC SN) subtype. We now know with a growing degree of confidence that solitary stars in the range of $\sim 8-16 M_{\odot}$ inevitably explode as Type II-plateau supernovae (SNe II-P; e.g., Smartt et al. 2009), with much of their hydrogen envelope still intact (see Filippenko 1997 for a discussion of SN classification). We also have evidence that the Type II-narrow (IIn) SN 2005gl had a luminous $\left(M_{V}=-10.3 \mathrm{mag}\right.$ ), very massive (possibly with initial mass $M_{\text {ini }}>50 M_{\odot}$ ) progenitor star that exploded while in the luminous blue variable phase (Gal-Yam \& Leonard 2009). This progenitor, the only example so far identified for an SN IIn, likely had a far smaller fraction of its outer $\mathrm{H}$ layer remaining than do SNe II-P. Various indirect clues may indicate that at least some of the Type $\mathrm{Ib} / \mathrm{c} \mathrm{SNe}$ are connected to the Wolf-Rayet phase, which is expected to occur for stars with $M_{\text {ini }}>25-30 M_{\odot}$ (e.g., Crowther 2007). That leaves the Type II-linear (II-L) SNe with no directly known progenitor star,

\footnotetext{
* Based in part on observations made with the NASA/ESA Hubble Space Telescope (HST), obtained from the Data Archive at the Space Telescope Science Institute, which is operated by the Association of Universities for Research in Astronomy (AURA), Inc., under NASA contract NAS 05-26555; the $6.5 \mathrm{~m}$ Magellan Clay Telescope located at Las Campanas Observatory, Chile; various telescopes at Lick Observatory; the $1.3 \mathrm{~m}$ PAIRITEL on Mt Hopkins; the SMARTS Consortium $1.3 \mathrm{~m}$ telescope located at Cerro Tololo Inter-American Observatory (CTIO), Chile; the $3.6 \mathrm{~m}$ Canada-France-Hawaii Telescope (CFHT), which is operated by the National Research Council of Canada, the Institut National des Sciences de l'Univers of the Centre National de la Recherche Scientifique of France, and the University of Hawaii; and the W. M. Keck Observatory, which is operated as a scientific partnership among the California Institute of Technology, the University of California, and NASA, with generous financial support from the W. M. Keck Foundation. 8 Clay Fellow.
}

as well as the range $M_{\mathrm{ini}}=18-30 M_{\odot}$ (exactly the range that Smartt et al. 2009 dubbed "the red supergiant (RSG) problem") without a well-established endpoint. From the rates derived with the Lick Observatory Supernova Search for a sample of wellstudied SNe (W. Li et al. 2010, in preparation), we know that the majority of massive stars end their lives as SNe II-P, while the incidences of SNe II-L and SNe IIn are comparatively rare ( $\sim 7 \%$ and $\sim 6 \%$ of all CC SNe, respectively).

Here, we examine the case of SN 2009kr in NGC 1832. SN 2009kr was discovered by Nakano \& Itagaki (2009) on 2009 November 6.73 (UT dates are used throughout) and was spectroscopically classified as an SN IIn (Tendulkar et al. 2009), and then as a "young type-II" SN (Steele et al. 2009). Li et al. (2009) first identified a possible progenitor star in archival Hubble Space Telescope (HST) images from 2004, using as reference a combined $160 \mathrm{~s} r^{\prime}$ image from CFHT+MegaCam ${ }^{9}$ on November 21.49. Here, we show that both early-time photometric and spectroscopic observations strongly suggest that this object is an SN II-L, and we increase our confidence in the progenitor identification via further comparison of the HST images with Keck-II/NIRC2 adaptive-optics (AO) data.

\section{THE EARLY-TIME NATURE OF SN 2009kr}

\subsection{Photometry}

Optical BVRI images of SN 2009kr were obtained with the Lick Observatory $0.76 \mathrm{~m}$ Katzman Automatic Imaging Telescope (KAIT; Filippenko et al. 2001) and the $1.0 \mathrm{~m}$ Nickel telescope, and SMARTS+ANDICAM at CTIO. They were all initially reduced using standard procedures (see, e.g., Poznanski

\footnotetext{
9 A joint project of CFHT and CEA/DAPNIA.
} 
et al. 2009). Because of our follow-up campaign on SN 2004gq (Modjaz 2007), also in NGC 1832, we had a well-calibrated photometric sequence in the host-galaxy field and template images in all passbands for image subtraction. We used an image-reduction pipeline (Ganeshalingam et al. 2010) to reduce all data and calibrate the photometry to the standard Johnson $B V$ and Cousins RI system.

Near-infrared (NIR) observations were obtained with the $1.3 \mathrm{~m}$ Peters Automated Infrared Imaging Telescope (PAIRITEL; Bloom et al. 2006) and reduced using standard procedures. We used archival Two Micron All Sky Survey (2MASS) images of NGC 1832 as templates to subtract from the SN images using HOTPANTS ${ }^{10}$ and calibrated the $J H K_{s}$ photometry against 2MASS stars in the field.

The $B V R I J H K_{\mathrm{s}}$ light curves are shown in Figure 1(a), relative to $B$-band maximum $(15.95 \pm 0.02 \mathrm{mag}$ on November $13 \pm 1$, or JD 2,455,149 \pm 1 ). For comparison, we also show the light curves of the SNe II-L 1979C (Balinskaia et al. 1980; de Vaucouleurs et al. 1981; Barbon et al. 1982b), 1980K (Buta 1982; Dwek et al. 1983), 1990K (Cappellaro et al. 1995), and 2001cy (unpublished KAIT data); the SNe II-P 1999em (Hamuy et al. 2001; Leonard et al. 2002) and 1992H (Clocchiatti et al. 1996); and, the possible SN II-L 2000dc (Poznanski et al. 2009). SN $2009 \mathrm{kr}$ does not follow the SN II-P plateau; instead, it more closely resembles the SN II-L linear decline (see Barbon et al. 1979). A steeper decline is present in VRI after $\sim 65$ days in the SN 2009kr light curves; however, this decline was also observed for SN $1980 \mathrm{~K}$ in $V$. From our adopted distance and total extinction (see Section 2.2), SN 2009kr reached $M\left(B_{\max }\right)=-16.48 \pm 0.30 \mathrm{mag}$, typical of SNe II-L (Young \& Branch 1989). We thus conclude that SN 2009kr displays the photometric behavior of an SN II-L.

\subsection{Spectroscopy}

Low-resolution spectra of SN 2009kr were obtained on 2009 November 10 and 25, December 9 and 18, and 2010 January 20 with the Lick $3 \mathrm{~m}$ Shane telescope+Kast spectrograph (Miller \& Stone 1993); on January 8 with the Clay telescope+MagE (Marshall et al. 2008); on February 15 with the 10 m Keck-II telescope+DEIMOS; and on March 9 with the 10 m Keck-I telescope+LRIS. The spectra were reduced and calibrated using standard procedures (e.g., Matheson et al. 2000). The observing conditions were not photometric, resulting in uncertain absoluteflux calibrations. We observed with the slit placed at the parallactic angle (Filippenko 1982).

We show the rest-frame spectral sequence in Figure 1(b); for comparison, we also display spectra of the SN II-L 1980K (Barbon et al. 1982a); the SNe II-P 1999em (Leonard et al. 2002), 1992H (Clocchiatti et al. 1996) and 2004et (Sahu et al. 2006); and the SN II 2001cy at similar epochs. Poznanski et al. (2009) rejected SN 2001cy from their SN II-P sample, implying that it likely may be an SN II-L. Indeed, the SN 2001cy light curve is very similar (but of inferior quality and coverage) to that of SN 2000dc shown in Figure 1(a). The following corrections for $E(B-V)$ tot have been made: SN 1999em (0.10 mag; Leonard et al. 2002), SN 1992H (0.09 mag; Clocchiatti et al. 1996), SN 2004et (0.41 mag; Maguire et al. 2010), SN 1980K (0.40 mag; Barbon et al. 1982a), and SN 2009kr (0.08 mag; see below). SN 2001cy has been corrected only for Galactic reddening, $E(B-V)_{\mathrm{Gal}}=0.21 \mathrm{mag}$ (Schlegel et al. 1998).

$\overline{10}$ http://www.astro.washington.edu/users/becker/hotpants.html
The spectral sequence for $\mathrm{SN} 2009 \mathrm{kr}$ is typical of many SNe II: the earliest spectra exhibit a blue continuum, with relatively weak spectral features, followed by the onset of increased Balmer-line emission, together with the emergence of P Cygni-like features. However, clearly SN 2009kr differs from the canonical SN 1999em or the peculiar SNe 1992H and 2004et; in particular, the SN 2009kr P Cygni $\mathrm{H} \alpha$ profile is dominated by the broad emission component. Several narrow emission lines appear superposed on the spectra, but these most likely originate from a neighboring $\mathrm{H}$ II region, seen $\sim 1^{\prime \prime}-2^{\prime \prime}$ northeast of the $\mathrm{SN}$ in an $\mathrm{H} \alpha$ image of the host galaxy (unpublished ESO archival data; the region's ionizing cluster can also be seen in the HST images). The SN 2009kr spectra more closely resemble those of SN $1980 \mathrm{~K}$ or SN 2001cy, which also exhibit relatively weak $\mathrm{H} \alpha$ absorption. The optical photometric decline rates $\left(\gtrsim 1 \mathrm{mag}(100 \mathrm{day})^{-1}\right)$ of SNe 1980K, 2001cy, and $2009 \mathrm{kr}$ suggest that they are all SNe II-L (see Section 2.1). Since spectroscopic features of SNe II appear to be correlated with their photometric behavior at early times (Schlegel 1996; Filippenko 1997), the similarities in the spectra of these three SNe are not unexpected.

We also obtained a high-resolution optical spectrum of SN 2009kr on 2009 November 25.46 with CFHT+ESPaDOnS, with four exposures of $833 \mathrm{~s}$ each, from which we confirm that the narrow $\mathrm{H} \alpha$ emission likely originates from the $\mathrm{H}$ II region. Moreover, we also used this spectrum to estimate the reddening toward SN $2009 \mathrm{kr}$ via measurement of the Na I D equivalent width $(\mathrm{EW})$ at the host-galaxy redshift $(z=0.006)$. We found $\mathrm{EW}(\mathrm{Na}$ I D1 $\lambda 5890)=0.044 \pm 0.003 \AA$ and $\mathrm{EW}(\mathrm{Na}$ I $\mathrm{D} 2 \lambda 5896)=0.032 \pm 0.004 \AA$. Using the relation between extinction and EW(Na I D) from N. Elias-Rosa et al. (2010, in preparation) and assuming the Cardelli et al. (1989) reddening law with updated wavelengths and a Galactic foreground $E(B-V)=0.07 \mathrm{mag}$ (Schlegel et al. 1998), we derive $E(B-V)_{\mathrm{tot}}=0.08 \pm 0.01 \mathrm{mag}\left(E(V-I)_{\mathrm{tot}}=0.11 \pm\right.$ $0.01 \mathrm{mag}$ ), which we adopt for the SN. This relatively low extinction is consistent with both the SN color comparison (Section 2.1) and the overall blue continua seen in the earlytime SN 2009kr spectra (Figure 1(b)).

\section{IDENTIFICATION OF THE PROGENITOR CANDIDATE}

Pairs of HST images of NGC 1832 were obtained with WFPC2 in bands F555W ( $\sim V ; 460 \mathrm{~s}$ total) and $\mathrm{F} 814 \mathrm{~W}$ ( $\sim$ I; 700 s total) on 2008 January 11 (program GO-10877, PI: W. Li), as SN 2004gq follow-up observations. The SN $2009 \mathrm{kr}$ site is located on the WF3 chip $\left(0^{\prime \prime} 1 \mathrm{pixel}^{-1}\right)$. Cosmic-ray hits were rejected, and a $1600 \times 1600$ pixel mosaic of all four WFPC2 chips was constructed using the STSDAS package routines crrej and wmosaic within IRAF. ${ }^{11}$ Li et al. (2009) were able to isolate the SN location in the WFPC2 images to 0.43 pixel $\left(0^{\prime} .043\right)$ through comparison with a ground-based, post-explosion CFHT+MegaCam image.

We were then able to better confirm the identification of this candidate through $K_{\mathrm{p}}$-band NIRC2 "wide" camera $\left(00^{\prime}\right.$.04 pixel $^{-1}, 40^{\prime \prime} \times 40^{\prime \prime}$ field of view $)$ images obtained on 2009 November 28 with Keck-II+AO. Each of the $10 \mathrm{~s}$ frames was sky subtracted using the median of the dithered exposures and

\footnotetext{
11 IRAF is distributed by the National Optical Astronomy Observatories, which are operated by the Association of Universities for Research in Astronomy, Inc., under cooperative agreement with the National Science Foundation.
} 

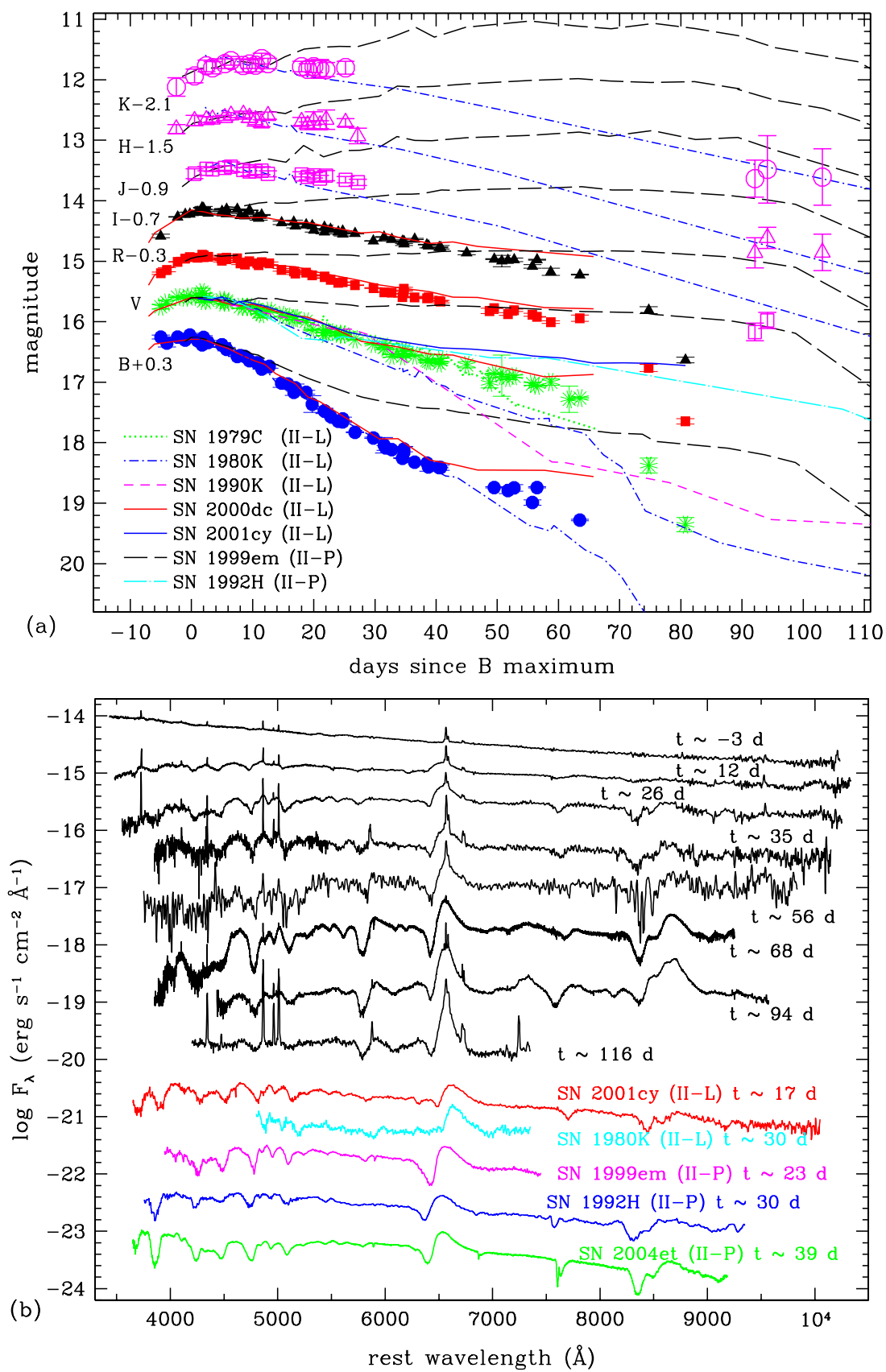

Figure 1. (a) SN 2009kr optical and NIR light curves, together with those of other SNe II-L and SNe II-P. The comparison light curves are adjusted in time and magnitude to match those of SN 2009kr. (b) SN 2009kr spectral sequence, along with comparison spectra of other SNe II-L and SNe II-P. All spectra have been corrected for their host-galaxy recession velocities and for reddening (see the text). Ages are relative to $B$ maximum light. The continuum of the $t \approx 116$ day spectrum of SN 2009kr appears to be significantly contaminated by light from the nearby star cluster.

then "shifted-and-added" using IRAF. They were also corrected for distortion. ${ }^{12}$

We achieved high-precision relative astrometry by geometrically transforming the pre-explosion images to match the post-explosion ones. We first "drizzled" 13 the pre-explosion images for each band to the higher NIRC2+AO resolution. Using 5-7 point-like sources in common between the two data sets and the IRAF tasks geomap and geotran, we carried out a geometrical transformation between the two sets of images. The positions (and their uncertainties) of the SN and the progenitor candidate are derived by averaging the measure-

\footnotetext{
12 http://www2.keck.hawaii.edu/inst/nirc2/forReDoc/post_observing/dewarp/

13 http://www.stsci.edu/hst/wfpc2/analysis/drizzle.html
}

ments from two centroiding methods, the task daofind within IRAF/DAOPHOT and imexamine within IRAF. The differences between the $\mathrm{SN}$ and the progenitor candidate positions, compared with the total estimated astrometric uncertainty, are given in Table 1. As an additional check, we transformed the NIRC2+AO image relative to only the WF3 chip image in F814W at its native resolution $\left(0^{\prime} .1\right.$ pixel $\left.^{-1}\right)$ and performed the registration. In this case, the positional difference is 19 mas, within $\sim 1 \sigma$ of the uncertainty in the astrometric solution. Note that no other source was located within a $5 \sigma$ radius from the progenitor candidate position identified by $\mathrm{Li}$ et al. (2009); the H II region to the northeast is located at $>25 \sigma$ (see Figure 2). 

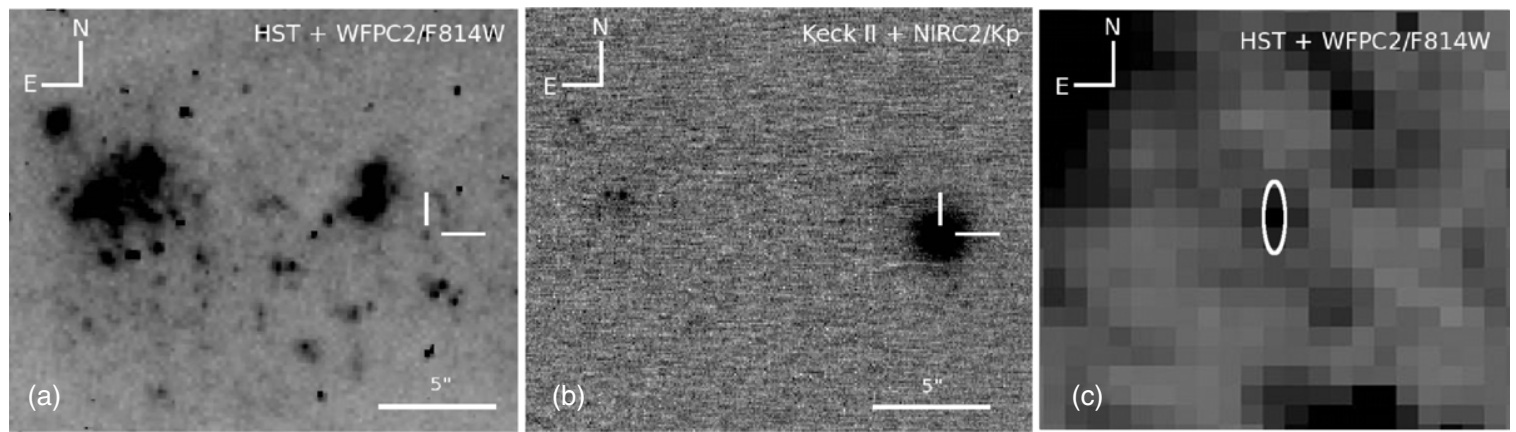

Figure 2. (a) Subsections of the pre-explosion $H S T+$ WFPC2/F814W images of NGC 1832 and (b) the post-explosion AO $K_{\mathrm{p}}$ image of SN $2009 \mathrm{kr}$ with Keck II+NIRC2. The approximate positions of the candidate progenitor and the SN are indicated by tick marks. (c) $5^{\prime \prime} \times 5^{\prime \prime}$ detail of the HST image. The $3 \sigma$ positional uncertainty ellipse is $0{ }^{\prime} .03 \times 00^{\prime} 09$ in radius (see Table 1 ).

Table 1

SN and Progenitor Candidate Position Comparison

\begin{tabular}{lcc}
\hline \hline \multicolumn{1}{c}{ Angular Quantity } & F555W $(\alpha / \delta)$ & $\mathrm{F} 814 \mathrm{~W}(\alpha / \delta)$ \\
\hline Uncertainty in the progenitor position (mas) & $0 / 2$ & $0 / 24$ \\
Uncertainty in the SN position (mas) & $8 / 13$ & $8 / 13$ \\
Geometric transformation (mas) & $4 / 15$ & $5 / 13$ \\
Total uncertainty (mas) & $9 / 20$ & $9 / 30$ \\
\hline Difference in position (mas) & $13 / 4$ & $7 / 22$ \\
\hline
\end{tabular}

Notes. Uncertainties $(1 \sigma)$ in the $\mathrm{SN}$ and progenitor candidate right ascension $(\alpha)$ and declination $(\delta)$ positions for each band, in milliarcsec (mas), were estimated as the standard deviation of the average measurements. Uncertainties in the geometric transformation were derived from the differences in the fiducial star positions before and after the transformation. The total uncertainty is the quadrature sum of all uncertainties. The last row lists the residual difference between the SN and progenitor position after the geometric transformation; see the text.

\section{THE NATURE OF THE PROGENITOR}

We also measured photometry of the HST images using HSTphot $^{14}$ (Dolphin 2000). The output from this package automatically includes the transformation from $\mathrm{F} 555 \mathrm{~W}$ and F814W to $V$ and $I$.

Adopting a distance modulus to NGC 1832 derived from the recession velocity corrected for Virgo infall (32.09 \pm 0.30 mag; this uncertainty arises from a possible $250 \mathrm{~km} \mathrm{~s}^{-1}$ peculiar velocity ${ }^{15}$ ) and the assumed $\mathrm{SN}$ extinction, we find that the absolute magnitudes of the progenitor candidate are $M_{V}^{0}=$ $-7.80 \pm 0.33$ and $M_{I}^{0}=-8.75 \pm 0.32$, entirely consistent with a highly luminous supergiant. The intrinsic color, $(V-I)_{0}=$ $0.95 \pm 0.21 \mathrm{mag}$, is significantly more "yellow" than the colors of normal RSGs (e.g., Drilling \& Landolt 2000).

We can directly determine the metallicity in the SN environment from the Canada-France-Hawaii Telescope (CFHT) high-resolution spectrum, by measuring the $\mathrm{H} \alpha$ and [N II] $\lambda 6584$ line intensities and applying the (Pettini \& Pagel 2004) cubic-fit relation between the ratio of these two lines and the oxygen abundance; no correction is made for the low extinction. Doing so, we find that $12+\log (\mathrm{O} / \mathrm{H})=8.67$. Given that the solar value is $8.69 \pm 0.05$ (Asplund et al. 2009), we consider it most likely that this environment has roughly solar metallicity.

The star's color corresponds to an effective temperature $T_{\text {eff }}=5300 \pm 500 \mathrm{~K}$ and a $V$ bolometric correction in the range

\footnotetext{
14 HSTphot is a stellar photometry package designed for use with WFPC2 images. We used v1.1.7b, updated 2008 July 19.

15 From NED, http://nedwww.ipac.caltech.edu/
}

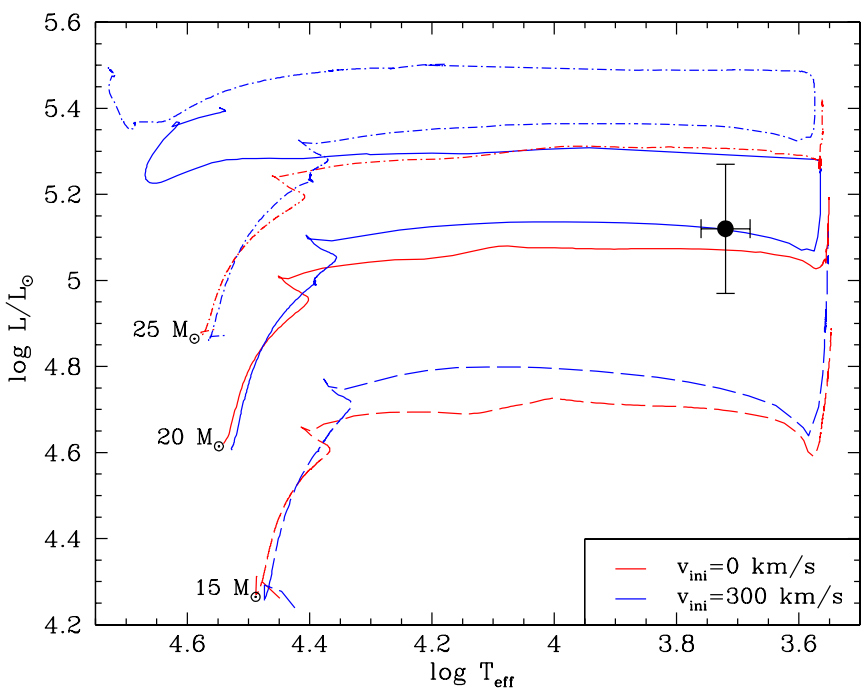

Figure 3. H-R diagram showing $L_{\mathrm{bol}}$ and $T_{\text {eff }}$ for the candidate progenitor of SN $2009 \mathrm{kr}$ (filled circle). Model stellar evolutionary tracks for solar metallicity (Hirschi et al. 2004) are also shown for a rotation of $v_{\text {ini }}=0 \mathrm{~km} \mathrm{~s}^{-1}$ (dotdashed, solid, and dashed red lines) and $v_{\text {ini }}=300 \mathrm{~km} \mathrm{~s}^{-1}$ (dot-dashed, solid, and dashed blue lines).

-0.47 to $-0.13 \mathrm{mag}$, for an assumed surface gravity $\log g=$ +0.5 (Kurucz Atlas 9 models, CD-ROMs 13, 18). This results in $L_{\text {bol }}=10^{(5.12 \pm 0.15)} L_{\odot}$ (assuming $M_{\text {bol }}(\odot)=4.74 \mathrm{mag}$ ). In Figure 3, we show a Hertzsprung-Russell (H-R) diagram including the progenitor candidate. In addition, we illustrate model evolutionary tracks (Hirschi et al. 2004) for stars with $M_{\text {ini }}=15,20$, and $25 M_{\odot}$, with rotation $\left(v_{\text {ini }}=300 \mathrm{~km} \mathrm{~s}^{-1}\right)$ and without rotation.

The location of the progenitor candidate in the H-R diagram is clearly not consistent with the $15 M_{\odot}$ tracks, an initial mass which lies within the range for SN II-P progenitors (Smartt et al. 2009). Unfortunately, the mass bins for these tracks are large (5 $M_{\odot}$ increments). However, taking into account the uncertainty in the progenitor candidate's luminosity, interpolating by eye between the tracks implies that $M_{\text {ini }} \approx 18-24 M_{\odot}$. We note that this is consistent with the upper limit of $<20 M_{\odot}$ on the SN 1980K progenitor (Smartt et al. 2009, using more current theoretical tracks) and the lower limit of $\gtrsim 17-18 M_{\odot}$ on the SN II-L 1979C progenitor (Van Dyk et al. 1999).

That such a massive progenitor would be somewhat bluer than would be expected for the normal RSG progenitors of SNe II-P is also consistent with the star being in a post-RSG phase. Such an expectation follows from the theoretical models by Hirschi et al. 


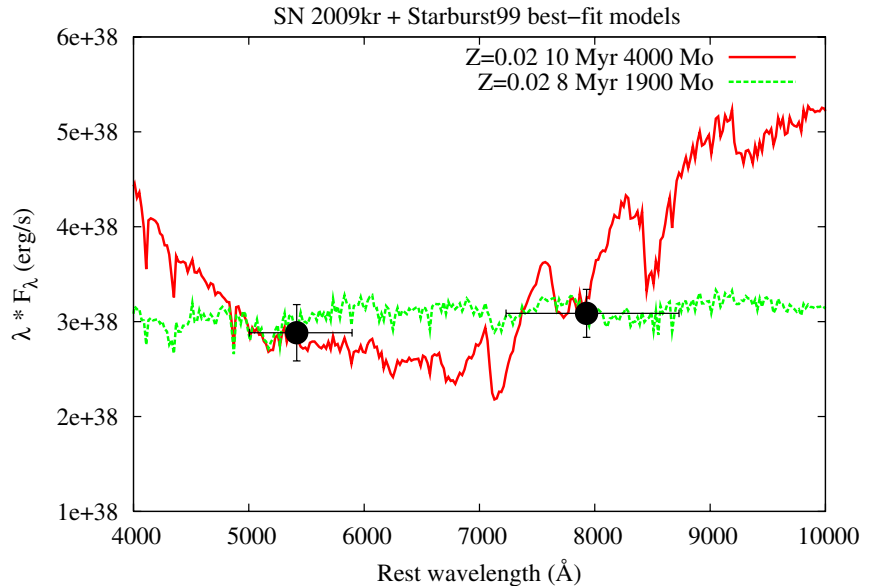

Figure 4. Comparison of the progenitor candidate (filled circles) with model spectral energy distributions for star clusters (Starburst99; Vazquez \& Leitherer 2005; lines) for solar metallicity. The model parameters (age and total mass) are indicated in the legend.

(2004) for rotating stars at higher masses; the rotating models are more luminous and evolve to the RSG phase before the ignition of He burning, which results in higher mass-loss rates, the loss of most of the $\mathrm{H}$ envelope before the termination of He burning, and evolution toward the blue before the terminal points. From Figure 3 one can see that rotation makes little difference in the evolution of a $15 M_{\odot}$ star; both the rotating and nonrotating tracks terminate as an RSG. However, for a $20 M_{\odot}$ star, rotation has a profound evolutionary effect. Though we cannot tell from these models what occurs for stars in the 15-20 $M_{\odot}$ range, it is reasonable to assume that rotation begins to affect those models nearing $20 M_{\odot}$. Thus, we might expect the progenitor candidate, based on our mass estimate above, to have evolved toward the blue before explosion.

Similar behavior has occurred for the post-RSG star IRC+10420, a mass-losing hypergiant that is transiting the socalled "yellow void" (Humphreys et al. 2002), although it is more luminous and hotter than the SN 2009kr progenitor candidate. Likely more analogous are the "anomalous" yellow supergiants in the Magellanic Clouds, which are in the mass range $15-20 M_{\odot}$ and show evidence for post-RSG evolution (Humphreys et al. 1991), albeit at subsolar metallicity.

Of course, there are caveats; as we found for the peculiar SN II-P 2008cn (Elias-Rosa et al. 2009), a yellow progenitor color could result from evolution in an interacting binary. Additionally, although the differences between the SN and progenitor candidate positions are practically within the total uncertainties, these are still large, and, given the host galaxy's distance (a single NIRC2 pixel corresponds to $\sim 5 \mathrm{pc}$ ), it is possible that we have not identified the SN progenitor at all, but rather a compact star cluster.

If so, adopting the $\mathrm{SN}$ extinction, we can fit the $V$ and $I$ fluxes for the progenitor candidate with the Starburst 99 code ${ }^{16}$ model spectral energy distributions and find an excellent fit with a 10 Myr track (Figure 4). If the SN progenitor were a member of this cluster, this age estimate would still be consistent with the lifespan of a $\sim 20 M_{\odot}$ star. The $8 \mathrm{Myr}$ model also provides a good fit. However, the lowest possible cluster luminosity that exceeds the luminosity of the most massive $8 \mathrm{Myr}$-old star is $M_{V} \approx-8.1 \mathrm{mag}$, and for $10 \mathrm{Myr}$ it is $M_{V} \approx-7.6 \mathrm{mag}$ (Cerviño $\&$ Luridiana 2004). Both of these are roughly consistent with

\footnotetext{
16 http://www.stsci.edu/science/starburst99/, Vazquez \& Leitherer (2005).
}

the candidate's luminosity. Furthermore, we would ordinarily expect compact star clusters to have $M_{V}<-8.6$ mag (Bastian et al. 2005; Crockett et al. 2008). Additional dust obscuration, beyond our assumed extinction, would imply that a putative cluster could be more luminous, but it would also be bluer and younger.

A similar study has been done contemporaneously by Fraser et al. (2010), identifying the same candidate progenitor. However, Fraser et al. (2010) suggest that the SN is a spectrally peculiar SN II-P, with only $r$-band photometry reported. Differences also exist in the metallicity estimate and theoretical models employed, leading to somewhat different estimates for $M_{\text {ini }}\left(15_{-4}^{+5} M_{\odot}\right.$ by Fraser et al. 2010).

\section{CONCLUSIONS}

Based on $\sim 3$ months of follow-up observations from discovery, we conclude that SN $2009 \mathrm{kr}$ is an SN II-L, a relatively rare subclass of CC SNe. We identified an object in pre-explosion $H S T$ images which agrees astrometrically with the SN $2009 \mathrm{kr}$ position, using NIR SN images obtained with NIRC2+AO on Keck. From the SN distance and extinction, we find that the object is consistent with a highly luminous supergiant star. Placing the inferred $L_{\text {bol }}$ and $T_{\text {eff }}$ of the progenitor candidate on an H-R diagram, we infer $M_{\text {ini }} \approx 18-24 M_{\odot}$. Its yellow color implies that the star may have exploded in a post-RSG evolutionary state, which is predicted by our assumed theoretical stellar models. If this star is the SN 2009kr progenitor, this would be the first-ever direct identification of an SN II-L progenitor. The star's mass estimate also may be a link between the upper mass range for SNe II-P (Smartt et al. 2009) and the estimated progenitor mass for SN IIn 2005gl (Gal-Yam \& Leonard 2009).

Ultimately, the definitive indication that we have identified the SN 2009kr progenitor is through very late-time imaging, such as with HST (Maund \& Smartt 2009). However, as an SN II-L, akin to the long-lasting SN 1979C (Milisavljevic et al. 2009), we may have to wait at least a decade to image the SN site using the James Webb Space Telescope.

We thank C. Blake, S. B. Cenko, B. Cobb, E. Falco, M. Kandrashoff, J. Kong, M. Modjaz, D. Starr, and T. Yuan for their assistance. J.V. received support from Hungarian OTKA Grant K76816, NSF Grant AST-0707769, and Texas Advanced Research Project grant ARP-0094. A.V.F.'s group and KAIT are supported by NSF grant AST-0908886, the Sylvia \& Jim Katzman Foundation, the TABASGO Foundation, and NASA through grants AR-11248 and GO-10877 from STScI. PAIRITEL is operated by SAO with support from the Harvard University Milton Fund, UC Berkeley, University of Virginia, and NASA/Swift grant NNX09AQ66G. J.S.B. and his group are partially funded by a DOE SciDAC grant.

Facilities: HST (WFPC2); KAIT; Nickel; Shane; CFHT(MegaCam); Keck:I(LRIS); Keck:II(NIRC2+AO, DEIMOS); CTIO:1.3m(SMARTS); Magellan:Clay(MagE)

\section{REFERENCES}

Asplund, M., Grevesse, N., Sauval, A. J., \& Scott, P. 2009, A\&A Rev., 47, 481 Balinskaia, I. S., Bychkov, K. V., \& Neizvestnyi, S. I. 1980, A\&A, 85, L19

Barbon, R., Ciatti, F., \& Rosino, L. 1979, A\&A, 72, 287

Barbon, R., Ciatti, F., \& Rosino, L. 1982a, A\&A, 116, 35

Barbon, R., Ciatti, F., Rosino, L., Ortolani, S., \& Rafanelli, P. 1982b, A\&A, 116,43

Bastian, N., Gieles, M., Efremov, Y. N., \& Lamers, H. J. G. L. M. 2005, A\&A, 443,79 
Bloom, J. S., et al. 2006, in ASP Conf. Ser. 331, Astronomical Data Analysis Software and Systems XV, ed. C. Gabriel et al. (San Francisco, CA: ASP), 751

Buta, R. J. 1982, PASP, 94, 578

Cappellaro, E., Danziger, I. J., Della Valle, M., Gouiffes, C., \& Turatto, M. 1995, A\&A, 293, 723

Cardelli, J. A., Clayton, G. C., \& Mathis, J. S. 1989, ApJ, 345, 245

Cerviño, M., \& Luridiana, V. 2004, A\&A, 413, 145

Clocchiatti, A., et al. 1996, AJ, 111, 1286

Crockett, R. M., et al. 2008, ApJ, 672, L99

Crowther, P. A. 2007, ARA\&A, 45, 177

de Vaucouleurs, G., de Vaucouleurs, A., Buta, R., Ables, H. D., \& Hewitt, A. V. 1981, PASP, 93, 36

Dolphin, A. E. 2000, PASP, 112, 1383

Drilling, J. S., \& Landolt, A. U. 2000, in Allen's Astrophysical Quantities, ed. A. N. Cox (New York: Springer), 381

Dwek, E., et al. 1983, ApJ, 274, 168

Elias-Rosa, N., et al. 2009, ApJ, 706, 1174

Filippenko, A. V. 1982, PASP, 94, 715

Filippenko, A. V. 1997, ARA\&A, 35, 309

Filippenko, A. V., Li, W., Treffers, R. R., \& Modjaz, M. 2001, in Small Telescope Astronomy on Global Scales, ed. W. P. Chen, C. Lemme, \& B. Paczyński (San Francisco, CA: ASP), 121

Fraser, M., et al. 2010, ApJ, submitted (arXiv:0912.2071)

Gal-Yam, A., \& Leonard, D. C. 2009, Nature, 458, 865

Ganeshalingam, M., et al. 2010, ApJS, submitted

Hamuy, M., et al. 2001, ApJ, 558, 615

Hirschi, R., Meynet, G., \& Maeder, A. 2004, A\&A, 425, 649
Humphreys, R. M., Davidson, K., \& Smith, N. 2002, AJ, 124, 1026

Humphreys, R. M., Kudritzki, R. P., \& Groth, H. G. 1991, A\&A, 245, 593

Leonard, D. C., et al. 2002, PASP, 114, 35

Li, W., et al. 2009, CBE Tel, 2042, 1

Maguire, K., et al. 2010, MNRAS, 284

Marshall, J. L., et al. 2008, Proc. SPIE, 7014, 169

Matheson, T., Filippenko, A. V., Ho, L. C., Barth, A. J., \& Leonard, D. C. 2000, AJ, 120, 1499

Maund, J. R., \& Smartt, S. J. 2009, Science, 324, 486

Milisavljevic, D., Fesen, R. A., Kirshner, R. P., \& Challis, P. 2009, ApJ, 692, 839

Miller, J. S., \& Stone, R. P. S. 1993, Lick Observatory Technical Report No. 66 (Santa Cruz, CA: Univ. California)

Modjaz, M. 2007, PhD thesis, Harvard University

Nakano, S., \& Itagaki, K. 2009, CBE Tel, 2006, 1

Pettini, M., \& Pagel, B. E. J. 2004, MNRAS, 348, 59

Poznanski, D., et al. 2009, ApJ, 694, 1067

Sahu, D. K., Anupama, G. C., Srividya, S., \& Muneer, S. 2006, MNRAS, 372, 1315

Schlegel, E. M. 1996, AJ, 111, 1660

Schlegel, D. J., Finkbeiner, D. P., \& Davis, M. 1998, ApJ, 500, 525

Smartt, S. J., et al. 2009, MNRAS, 395, 1409

Steele, T. N., et al. 2009, CBE Tel, 2011, 1

Tendulkar, S. P., et al. 2009, ATel, 2291, 1

Van Dyk, S. D., et al. 1999, PASP, 111, 313

Vazquez, G. A., \& Leitherer, C. 2005, ApJ, 621, 695

Young, T. R., \& Branch, D. 1989, ApJ, 342, L79 